\title{
Cut Limits on Hyperbolic Extensions
}

\author{
Pedro Ontaneda*
}

\begin{abstract}
Hyperbolic extensions were defined and studied in [4. Cut limits of families of metrics were introduced in [5]. In this paper we show that if a family of metrics $\left\{h_{\lambda}\right\}$ has cut limits then the family of hyperbolic extensions $\left\{\mathcal{E}_{k}\left(h_{\lambda}\right)\right\}$ also has cut limits.

The results in this paper are used in the problem of smoothing Charney-Davis strict hyperbolizations [2], [3].
\end{abstract}

\section{Section 1. Introduction.}

This paper deals with the relationship between two concepts: "hyperbolic extensions", which were studied in [4], and "cut limits of families of metrics", which were defined in [5]. Before stating our main result we first introduce these concepts here.

A. Hyperbolic extensions. Recall that the hyperbolic $n$-space $\mathbb{H}^{n}$ is isometric to $\mathbb{H}^{k} \times \mathbb{H}^{n-k}$ with warp product metric $\left(\cosh ^{2} r\right) \sigma_{\mathbb{H} k}+\sigma_{\mathbb{H}^{n-k}}$, where $\sigma_{\mathbb{H} l}$ denotes the hyperbolic metric of $\mathbb{H}^{l}$, and $r: \mathbb{H}^{n-k} \rightarrow[0, \infty)$ is the distance to a fixed point in $\mathbb{H}^{n-k}$. For instance, in the case $n=2$, since $\mathbb{H}^{1}=\mathbb{R}^{1}$ we have that $\mathbb{H}^{2}$ is isometric to $\mathbb{R}^{2}=\{(u, v)\}$ with metric $\cosh ^{2} v d u^{2}+d v^{2}$. The concept of "hyperbolic extension" is a generalization of this construction; we explain this in the next paragraph.

Let $\left(M^{n}, h\right)$ be a complete Riemannian manifold with center $o=o_{M} \in M$, that is, the exponential map $\exp _{o}: T_{o} M \rightarrow M$ is a diffeomorphism. The warp product metric

$$
f=\left(\cosh ^{2} r\right) \sigma_{\mathbb{H} k}+h .
$$

on $\mathbb{H}^{k} \times M$ is the hyperbolic extension (of dimension $k$ ) of the metric $h$. Here $r$ is the distance-to-o function on $M$. We write $\mathcal{E}_{k}(M)=\left(\mathbb{H}^{k} \times M, f\right)$, and $f=\mathcal{E}_{k}(h)$. We also say that $\mathcal{E}_{k}(M)$ is the hyperbolic extension (of dimension $k$ ) of $(M, h)$ (or just of $M$ ). Hence, for instance, we have $\mathcal{E}_{k}\left(\mathbb{H}^{l}\right)=\mathbb{H}^{k+l}$. Also write $\mathbb{H}^{k}=\mathbb{H}^{k} \times\left\{o_{M}\right\} \subset \mathcal{E}_{k}(M)$ and we have that any $p \in \mathbb{H}^{k}$ is a center of $\mathcal{E}_{k}(M)$ (see Remark $2.3(3)$ ).

\section{Remarks 1.1.}

1. Let $M^{n}$ have center $o$. Using a fixed orthonormal basis on $T_{o} M$ and the exponential map we can identify $M$ with $\mathbb{R}^{n}$, and $M-\{o\}$ with $\mathbb{R}^{n}-\{0\}=\mathbb{S}^{n-1} \times(0, \infty)$. Hence the spheres $\mathbb{S}^{n-1} \times\{r\} \subset \mathbb{S}^{n-1} \times(0, \infty)$ are geodesic spheres, and the rays $t \mapsto t v=(v, t) \in \mathbb{S}^{n-1} \times(0, \infty)=$

\footnotetext{
${ }^{*}$ The author was partially supported by a NSF grant.
} 
$M-\{o\}$, are distance minimizing geodesic rays emanating from the center.

2. Let $g^{\prime}$ be another metric on $M$. Suppose we can write $g^{\prime}=g_{r}^{\prime}+d r^{2}$ on $\mathbb{S}^{n-1} \times(0, \infty)=M-\{o\}$, (this last identification is done using $g$ ). Then the geodesic spheres around $o$, and the geodesics emanating from $o$ for $g$ and $g^{\prime}$ coincide.

B. Cut limits. Before we talk about "cut limits" we need some preliminary definitions and facts. Let $\left(M^{n}, g\right)$ have center $o$. Then the metric $g$ (outside the center) has the form $g=g_{r}+d r^{2}$. Here we are identifying (see 1.1) the space $M-\{o\}$ with $\mathbb{S}^{n-1} \times(0, \infty)$, thus each $g_{r}$ is a metric on the sphere $\mathbb{S}^{n-1}$.

\section{Examples.}

1. The Euclidean metric $\sigma_{\mathbb{R}^{n}}$ on $\mathbb{R}^{n}$ can be written as $\sigma_{\mathbb{R}^{n}}=g_{r}+d r^{2}$ with $g_{r}=r^{2} \sigma_{\mathbb{S}^{n-1}}$, where $\sigma_{\mathbb{S}^{n-1}}$ is the round metric on the sphere $\mathbb{S}^{n-1}$.

2. The hyperbolic metric $\sigma_{\mathbb{H}^{n}}$ on $\mathbb{R}^{n}$ can be written as $\sigma_{\mathbb{H}^{n}}=g_{r}+d r^{2}$ with $g_{r}=\sinh ^{2}(r) \sigma_{\mathbb{S}^{n-1}}$.

Let $(M, g)$ have center $o$ and write $g=g_{r}+d r^{2}$. Let $r_{0}>0$. We can think of the metric $g_{r_{0}}$ as being obtained from $g=g_{r}+d r^{2}$ by "cutting" $g$ along the sphere of radius $r_{0}$, so we call the metric $g_{r_{0}}$ on $\mathbb{S}^{n-1}$ the spherical cut of $g$ at $r_{0}$. Let

$$
\hat{g}_{r_{0}}=\left(\frac{1}{\sinh ^{2}\left(r_{0}\right)}\right) g_{r_{0}} .
$$

We call the metric $\hat{g}_{r_{0}}$ on $\mathbb{S}^{n-1}$ given by (1.2) the normalized spherical cut of $g$ at $r_{0}$. In the particular case that $g=g_{r}+d r^{2}$ is a warped-by-sinh metric we have $g_{r}=\sinh ^{2}(r) g^{\prime}$ for some fixed $g^{\prime}$ independent of $r$. In this case the spherical cut of $g=\sinh ^{2}(r) g^{\prime}+d t^{2}$ at $r_{0}$ is $\sinh ^{2}\left(r_{0}\right) g^{\prime}$, and the the normalized spherical cut at $r_{0}$ is $\hat{g}_{r_{0}}=g^{\prime}$.

Example. If $g=\sigma_{\mathbb{H}^{n}}=\sinh ^{2}(r) \sigma_{\mathbb{S}^{n-1}}+d r^{2}$, the normalized spherical cut at $r_{0}$ is $\left(\widehat{\sigma_{\mathbb{H}^{n}}}\right)_{r_{0}}=\sigma_{\mathbb{S}^{n-1}}$. And the spherical cut at $r_{0}$ is $\sinh ^{2}\left(r_{0}\right) \sigma_{\mathbb{S}^{n-1}}$.

Let $\left(M^{n}, g\right)$ have center $o$. We now consider families of metrics $\left\{g_{\lambda}\right\}_{\lambda>\lambda_{0}}$ on $M$ of the form $g_{\lambda}=\left(g_{\lambda}\right)_{r}+d r^{2}$. Here $\lambda_{0}>0$, and the identification $M-\{o\}=\mathbb{S}^{n-1} \times(0, \infty)$ is done using $g$; see Remark 1.1. We call such a family an $\odot$-family of metrics on $(M, g)$. (We use the symbol $\odot$ to evoke the idea that all metrics $g_{\lambda}$ have a common center and spheres). The reason we are interested in these families is that they are key ingredients in Riemannian Hyperbolization [3] (also see [5]). Moreover, the Main Theorem in this paper is used in [3].

Let $b \in \mathbb{R}$. By cutting each $g_{\lambda}$ at $b+\lambda$ we obtain a one-parameter family $\left\{\widehat{\left(g_{\lambda}\right)}\right\}_{\lambda+b}$ of metrics on the sphere $\mathbb{S}^{n-1}$. (The metric $\widehat{\left(g_{\lambda}\right)}{ }_{\lambda+b}$ is the normalized spherical cut of $g_{\lambda}$ at $\lambda+b$ ). Here $\lambda>\max \left\{\lambda_{0},-b\right\}$, so that the definition makes sense. We say that the $\left\{g_{\lambda}\right\}$ has cut limit at $b$ if this family $C^{2}$-converges, as $\lambda \rightarrow \infty$. That is, there is a $C^{2}$ metric $\hat{g}_{\infty}^{b}$ on $\mathbb{S}^{n-1}$ such that

$$
\left|{\widehat{\left(g_{\lambda}\right)}}_{\lambda+b}-\hat{g}_{\infty}^{b}\right|_{C^{2}\left(\mathbb{S}^{n-1}\right)} \longrightarrow 0 \quad \text { as } \quad \lambda \rightarrow \infty .
$$

Here the arrow means convergence in the $C^{2}$-norm on the space of $C^{2}$ metrics on $\mathbb{S}^{n-1}$. 
Remark 1.4. The $C^{2}$ norm is taken with respect to a fixed locally finite atlas with extendable charts, i.e. charts that can be extended to the (compact) closure of their domains.

Let $I \subset \mathbb{R}$ be an interval (compact or noncompact). We say that the $\odot$-family $\left\{g_{\lambda}\right\}$ has cut limits on $I$ if the convergence in (1.3) is uniform with compact supports in the variable in $b \in I$. Explicitly this means: for every $\epsilon>0$, and compact $K \subset I$ there is $\lambda_{*}$ such that $\left|{\widehat{\left(g_{\lambda}\right)}}_{\lambda+b^{\prime}}-\hat{g}_{\infty+b^{\prime}}\right|_{C^{2}\left(\mathbb{S}^{n-1}\right)}<\epsilon$, for $\lambda>\lambda_{*}$ and $b^{\prime} \in K$.

Remark 1.5. Equivalently, the $\odot$-family $\left\{g_{\lambda}\right\}$ has cut limits on $I$ if for every $\epsilon>0$, and $b \in I$ there are $\lambda_{*}$ and neighborhood $U$ of $b$ in $I$ such that $\left|\widehat{\left(g_{\lambda}\right)}{ }_{\lambda+b^{\prime}}-\hat{g}_{\infty+b^{\prime}}\right|_{C^{2}\left(\mathbb{S}^{n-1}\right)}<\epsilon$, for $\lambda>\lambda_{*}$ and $b^{\prime} \in U$.

If $\left\{g_{\lambda}\right\}$ has cut limits on $I$ then it has a cut limit at $b$, for every $b \in I$. Finally we say that $\left\{g_{\lambda}\right\}$ has a cut limits if $\left\{g_{\lambda}\right\}$ has a cut limits on $\mathbb{R}$.

Remark 1.6. If $\left\{g_{\lambda}\right\}_{\lambda}$ is a family of metrics then $\left\{g_{\lambda_{\left(\lambda^{\prime}\right)}}\right\}_{\lambda^{\prime}}$ is a reparametrization of $\left\{g_{\lambda}\right\}_{\lambda}$, where $\lambda^{\prime} \mapsto \lambda\left(\lambda^{\prime}\right)$ is a change of variables. For instance, if we use translations, the following holds: $\left\{g_{\lambda}\right\}_{\lambda}$ has cut limits at $b$ if and only if $\left\{g_{\lambda^{\prime}+a}\right\}_{\lambda^{\prime}}$ has cut limits at $b+a$; here the change of variables is $\lambda=\lambda^{\prime}+a$.

C. Statement of main result. Here is a natural question:

Question. If $\left\{h_{\lambda}\right\}_{\lambda}$ has cut limits, does $\left\{\mathcal{E}_{k}\left(h_{\lambda}\right)\right\}_{\lambda}$ have cut limits?

Remark. More generally we can ask whether $\left\{\mathcal{E}_{k}\left(h_{\lambda}\right)\right\}_{\lambda^{\prime}}$ has cut limits, where $\lambda=\lambda\left(\lambda^{\prime}\right)$. Of course the answer would depend on the change of variables $\lambda=\lambda\left(\lambda^{\prime}\right)$.

Our main result gives an affirmative answer to this question provided the family $\left\{h_{\lambda}\right\}$ is, in some sense, nice near the origin. Explicitly, we say that $\left\{h_{\lambda}\right\}_{\lambda>\lambda_{0}}$ is hyperbolic around the origin if there is a $B \in \mathbb{R}$ such that

$$
\widehat{\left(h_{\lambda}\right)_{\lambda+b}}=\sigma_{\mathbb{S}^{n-1}} .
$$

for every $b \leq B$ and every $\lambda>\max \left\{\lambda_{0},-b\right\}$. Note that this implies that each $h_{\lambda}$ is canonically hyperbolic on the ball of radius $\lambda+B$, i.e. $h_{\lambda}=\sinh ^{2}(r) \sigma_{\mathbb{S}_{n-1}}+d r^{2}$ on the ball of radius $\lambda+B$. Examples of $\odot$-families that are hyperbolic around the origin are families obtained using hyperbolic forcing [5].

As mentioned before our main result answers affirmatively the question above. Moreover it also says that some reparametrized families $\left\{\mathcal{E}_{k}\left(h_{\lambda}\right)\right\}_{\lambda^{\prime}}$ have cut limits as well, for certain change of variables $\lambda=\lambda\left(\lambda^{\prime}\right)$. Write $\lambda=\lambda\left(\lambda^{\prime}, \theta\right)=\sinh ^{-1}\left(\sinh \left(\lambda^{\prime}\right) \sin \theta\right)$, for a fixed $\theta$. We say that $\left\{\mathcal{E}_{k}\left(h_{\lambda}\right)\right\}_{\lambda^{\prime}}$ is the $\theta$-reparametrization of $\left\{\mathcal{E}_{k}\left(h_{\lambda}\right)\right\}_{\lambda}$. Note that if we consider an hyperbolic right triangle with one angle equal to $\theta$ and side (opposite to $\theta$ ) of length $\lambda$, then $\lambda^{\prime}$ is the length of the hypotenuse of the triangle. All $\theta$-reparametrizations, in the limit $\lambda^{\prime} \rightarrow \infty$, differ just by translations; that is, a simple calculation shows that $\lim _{\lambda^{\prime} \rightarrow \infty} \lambda\left(\lambda^{\prime}\right)-\lambda^{\prime}=\ln \sin \theta$. We are now ready to state our Main result. 
Main Theorem. Let $M$ have center o. Let $\left\{h_{\lambda}\right\}_{\lambda}$ be $\odot$-family of metrics on $M$. If $\left\{h_{\lambda}\right\}_{\lambda}$ is hyperbolic around the origin and has cut limits, then for every $\theta \in(0, \pi / 2]$ the $\theta$-reparametrization $\left\{\mathcal{E}_{k}\left(h_{\lambda}\right)\right\}_{\lambda^{\prime}}$ has cut limits.

Note that $\theta=\pi / 2$ gives $\lambda=\lambda^{\prime}$ answering the question above. The paper is structured as follows. In Section 2 we review some facts about hyperbolic extensions. In Section 3 we introduce useful coordinates on the spheres of a hyperbolic extension. In Section 4 we study normalized spherical cuts on hyperbolic extensions. Finally in Section 5 we deal with cut limits in a bit more detail and prove the Main Theorem.

\section{Section 2. Hyperbolic Extensions.}

Notational convention: we will denote all fixed centers on manifolds by the same letter " $O$ ". If the manifold $M$ needs to be specified we will write $o=o_{M}$, which means that $o$ is a center in $M$.

Note that $\mathbb{H}^{k}$ is convex in $\mathcal{E}_{k}(M)$ (see [1, p.23). Let $\eta$ be a complete geodesic in $M$ passing though $o$ and let $\eta^{+}$be one of its two geodesic rays (beginning at $o$ ). Then $\eta$ is a totally geodesic subspace of $M$ and $\eta^{+}$is convex (see [4]). Also, let $\gamma$ be a complete geodesic in $\mathbb{H}^{k}$. The following two results are proved in Section 3 of [4].

Lemma 2.1. The subspace $\gamma \times \eta^{+}$is a convex in $\mathcal{E}_{k}(M)$, and $\gamma \times \eta$ is totally geodesic in $\mathcal{E}_{k}(M)$.

Corollary 2.2. The subspaces $\mathbb{H}^{k} \times \eta^{+}$and $\gamma \times M$ are convex in $\mathcal{E}_{k}(M)$. Also $\mathbb{H}^{k} \times \eta$ is totally geodesic in $\mathcal{E}_{k}(M)$.

\section{Remarks 2.3.}

1. By convexity above we mean the following: a set $A$ is convex if given two points in $A$ any distance minimizing geodesic joining these points lies in $A$.

2. As pointed out in Section 3 of [4], the proof of Lemma 2.1 (which is Lemma 3.1 in [4]) can easily be adapted to show that $\{y\} \times M$ are convex in $\mathcal{E}_{k}(M)$. Alternatively, it is not hard to prove that $\{y\} \times M$ is convex in $\gamma \times M$; this together with Corollary 3.2 implies that $\{y\} \times M$ are convex in $\mathcal{E}_{k}(M)$.

3. Note that $\mathbb{H}^{k} \times \eta$ (with metric induced by $\mathcal{E}_{k}(M)$ ) is isometric to $\mathbb{H}^{k} \times \mathbb{R}$ with warp product metric $\cosh ^{2} v \sigma_{\mathbb{H} k}+d v^{2}$, which is just hyperbolic $(k+1)$-space $\mathbb{H}^{k+1}$. Also $\gamma \times \eta$ is isometric to $\mathbb{R} \times \mathbb{R}$ with warp product metric $\cosh ^{2} v d u^{2}+d v^{2}$, which is just hyperbolic 2-space $\mathbb{H}^{2}$. In particular every point in $\mathbb{H}^{k}=\mathbb{H}^{k} \times\{o\} \subset \mathcal{E}_{k}(M)$ is a center point.

As before we use $h$ to identify $M-\{o\}$ with $\mathbb{S}^{n-1} \times \mathbb{R}^{+}$. Sometimes we will denote a point $v=(u, r) \in \mathbb{S}^{n-1} \times \mathbb{R}^{+}=M-\{o\}$ by $v=r u$. Fix a center $o \in \mathbb{H}^{k} \in \mathcal{E}_{k}(M)$. Then, for $y \in \mathbb{H}^{k}-\{o\}$ we can also write $y=t w,(w, t) \in \mathbb{S}^{k-1} \times \mathbb{R}^{+}$. Similarly, using the exponential map we can identify $\mathcal{E}_{k}(M)-\{o\}$ with $\mathbb{S}^{k+n-1} \times \mathbb{R}^{+}$, and for $p \in \mathcal{E}_{k}(M)-\{o\}$ we can write $p=s x$, $(x, s) \in \mathbb{S}^{k+n-1} \times \mathbb{R}^{+}$.

We denote the metric on $\mathcal{E}_{k}(M)$ by $f$ and we can write $f=f_{s}+d s^{2}$. Since $\mathbb{H}^{k}$ is convex in $\mathcal{E}_{k}(M)$ we can write $\mathbb{H}^{k}-\{o\}=\mathbb{S}^{k-1} \times \mathbb{R}^{+} \subset \mathbb{S}^{k+n-1} \times \mathbb{R}^{+}$and $\mathbb{S}^{k-1} \subset \mathbb{S}^{k+n-1}$.

A point $p \in \mathcal{E}_{k}(M)-\mathbb{H}^{k}$ has two sets of coordinates: the polar coordinates $(x, s)=(x(p), s(p)) \in$ 
$\mathbb{S}^{k+n-1} \times \mathbb{R}^{+}$and the hyperbolic extension coordinates $(y, v)=(y(p), v(p)) \in \mathbb{H}^{k} \times M$. Write $M_{o}=\{o\} \times M$. Therefore we have the following functions:

$\begin{array}{lll}\text { the distance to } o \text { function: } & s: \mathcal{E}_{k}(M) \rightarrow[0, \infty), & s(p)=d_{\mathcal{E}_{k}(M)}(p, o) \\ \text { the direction of } p \text { function: } & x: \mathcal{E}_{k}(M)-\{o\} \rightarrow \mathbb{S}^{n+k-1}, & p=s(p) x(p) \\ \text { the distance to } \mathbb{H}^{k} \text { function: } & r: \mathcal{E}_{k}(M) \rightarrow[0, \infty), & r(p)=d_{\mathcal{E}_{k}(M)}\left(p, \mathbb{H}^{k}\right) \\ \text { the projection on } \mathbb{H}^{k} \text { function: } & y: \mathcal{E}_{k}(M) \rightarrow \mathbb{H}^{k}, & \\ \text { the projection on } M \text { function: } & v: \mathcal{E}_{k}(M) \rightarrow M, & \\ \text { the projection on } \mathbb{S}^{n-1} \text { function: } & u: \mathcal{E}_{k}(M)-\mathbb{H}^{k} \rightarrow \mathbb{S}^{n-1}, & v(p)=r(p) u(p) \\ \text { the length of } y \text { function: } & t: \mathcal{E}_{k}(M) \rightarrow[0, \infty), & t(p)=d_{\mathbb{H}^{k}}(y(p), o) \\ \text { the direction of } y \text { function: } & w: \mathcal{E}_{k}(M)-M_{o} \rightarrow \mathbb{S}^{k-1}, & y(p)=t(p) w(p)\end{array}$

Note that $r=d_{M}(v, o)$. Note also that, by Lemma 2.1, the functions $w$ and $u$ are constant on geodesics emanating from $o \in \mathcal{E}_{k}(M)$, that is $w(s x)=w(x)$ and $u(s x)=u(x)$.

Let $\partial_{r}$ and $\partial_{s}$ be the gradient vector fields of $r$ and $s$, respectively. Since the $M$-fibers $M_{y}=\{y\} \times M$ are convex the vectors $\partial_{r}$ are the velocity vectors of the speed one geodesics of the form $a \mapsto(y, a u), u \in \mathbb{S}^{n-1} \subset M$. These geodesics emanate from (and orthogonally to) $\mathbb{H}^{k} \subset \mathcal{E}_{k}(M)$. Also the vectors $\partial_{s}$ are the velocity vectors of the speed one geodesics emanating from $o \in \mathcal{E}_{k}(M)$. For $p \in \mathcal{E}_{k}(M)$, denote by $\triangle=\triangle(p)$ the right triangle with vertices $o$, $y=y(p), p$ and sides the geodesic segments $[o, p] \in \mathcal{E}_{k}(M),[o, y] \in \mathbb{H}^{k},[p, y] \in\{y\} \times M \subset \mathcal{E}_{k}(M)$. (These geodesic segments are unique and well-defined because: (1) $\mathbb{H}^{k}$ is convex in $\mathcal{E}_{k}(M)$, (2) $(y, o)=o_{\{y\} \times M}$ and $o$ are centers in $\{y\} \times M$ and $\mathbb{H}^{k} \subset \mathcal{E}_{k}(M)$, respectively. $)$

Let $\alpha: \mathcal{E}_{k}(M)-\mathbb{H}^{k} \rightarrow \mathbb{R}$ be the angle between $\partial_{s}$ and $\partial_{r}$ (in that order), thus cos $\alpha=f\left(\partial_{r}, \partial_{s}\right)$, $\alpha \in[0, \pi]$. Then $\alpha=\alpha(p)$ is the interior angle, at $p=(y, v)$, of the right triangle $\triangle=\triangle(p)$. We call $\beta(p)$ the interior angle of this triangle at $o$, that is $\beta(p)=\beta(x)$ is the spherical distance between $x \in \mathbb{S}^{k+n-1}$ and the totally geodesic sub-sphere $\mathbb{S}^{k-1}$. Alternatively, $\beta$ is the angle between the geodesic segment $[o, p] \subset \mathcal{E}_{k}(M)$ and the convex submanifold $\mathbb{H}^{k}$. Therefore $\beta$ is constant on each geodesic emanating from $o \in \mathcal{E}_{k}(M)$, that is $\beta(s x)=\beta(x)$. The following corollary follows from Lemma 2.1 (see 3.1 in [4]).

Corollary 2.4. Let $\eta^{+}$(or $\eta$ ) be a geodesic ray (line) in $M$ through o containing $v=v(p)$ and $\gamma$ a geodesic line in $\mathbb{H}^{k}$ through o containing $y=y(p)$. Then $\triangle(p) \subset \gamma \times \eta^{+} \subset \gamma \times \eta$.

Remark 2.5. Note that the right geodesic triangle $\triangle(p)$ has sides of length $r=r(p), t=t(p)$ and $s=s(p)$. By Lemma 2.1 and Remark 2.3(3) we can consider $\triangle$ as contained in a totally geodesic copy of hyperbolic 2-space $\mathbb{H}^{2}(p)$. The plane $\mathbb{H}^{2}(p)$ is well defined for $p$ outside $\mathbb{H}^{k} \cup(\{o\} \times M)$. We will write $\mathbb{H}^{2}(p)=\gamma_{w} \times \eta_{u}$, where $p=(y, v) \in \mathbb{H}^{k} \times M, y=t w, v=r u$.

Hence, by Remark 2.5, using hyperbolic trigonometric identities we can find relations between $r, t, s, \alpha$ and $\beta$. For instance, using the hyperbolic law of sines we get: 


$$
\sinh (r)=\sin (\beta) \sinh (s)
$$

In Section 4 we will need the following result.

Proposition 2.7. The following identity holds outside $\mathbb{H}^{k} \cup(\{o\} \times M)$

$$
\left(\sinh ^{2}(s)\right) d \beta^{2}+d s^{2}=\cosh ^{2}(r) d t^{2}+d r^{2}
$$

Proof. First a particular case. Take $M=\mathbb{R}$ and $k=1$, hence $\mathcal{E}_{k}(M)=\mathcal{E}_{1}(\mathbb{R})=\mathbb{H}^{2}$. In this case the left-hand side of the identity above is the expression of the metric of $\mathbb{H}^{2}$ in polar coordinates $(\beta, s)$, and right hand side of the equation is the expression of the same metric in the hyperbolic extension coordinates $(r, t)=(v, y)$. (Here $r$ and $t$ are "signed" distances.) Hence the equation holds in this particular case. This particular case, together with the fact that $\mathbb{H}^{2}(p)$ is isometric to $\mathbb{H}^{2}$, and the following claim prove the proposition.

Claim. The functionals $d \beta$, ds, $d t$, dr, at $p \in \mathbb{H}^{k} \cup(\{o\} \times M)$, are zero on vectors perpendicular to $\mathbb{H}^{2}(p)$.

Proof of the Claim. To prove the claim let $u$ be a vector perpendicular to $\mathbb{H}^{2}(p)$, at $p$. Since the ray $s \mapsto s x(p)$ is contained in $\mathbb{H}^{2}(p)$ we have that $u$ is tangent to the sphere of radius $s(p)$ centered at $o$. Therefore $d s(u)=0$.

Next we prove that $d r(u)=0$ and $d t(u)=0$. Note that $u$ is a linear combination of vectors perpendicular to $\mathbb{H}^{2}(p)$ that are either tangent to $\{y\} \times M$ or $\mathbb{H}^{k} \times\{v\}$, where $y=y(p)$ and $v=v(p)$. Therefore it is enough to assume $u$ is tangent to $\{y\} \times M$ or $\mathbb{H}^{k} \times\{v\}$.

First assume that $u$ is perpendicular to $\mathbb{H}^{2}(p)$ and tangent to $\{y\} \times M$. Since $u$ is tangent to $\{y\} \times M$ we get that $d t(u)$. And since $u$ is perpendicular to the ray $r \mapsto r v$ in $\{y\} \times M$ (because this ray is contained in $\left.\mathbb{H}^{2}(p)\right)$ we get that $d r(u)=0$.

Next assume that $u$ is perpendicular to $\mathbb{H}^{2}(p)$ and tangent to $\mathbb{H}^{k} \times\{v\}$. Then $d r(u)=0$. And since $u$ is perpendicular to the ray $t \mapsto t y$ in $\mathbb{H}^{k} \times\{v\}$ (because this ray is contained in $\mathbb{H}^{2}(p)$ ) we get that $d t(u)=0$.

Finally, the equation $d \beta(u)=0$ follows from $d s(u)=0, d t(u)=0, d r(u)=0$, the fact that $\beta$ is a function of $s, t, r$, and the chain rule. This proves the claim and concludes the proof of Proposition 2.7.

\section{Section 3. Coordinates On The Spheres $S_{s}\left(\mathcal{E}_{k}(M)\right)$.}

Let $N^{n}$ have center $o$. The geodesic sphere of radius $r$ centered at $o$ will be denoted by $\mathbb{S}_{r}=\mathbb{S}_{r}(N)$ and we can identify $\mathbb{S}_{r}$ with $\mathbb{S}^{n-1} \times\{r\}$.

Let $M$ have center $o$ and metric $h$. Consider the hyperbolic extension $\mathcal{E}_{k}(M)$ of $M$ with center $o \in \mathbb{H}^{k}=\mathbb{H}^{k} \times\{o\} \subset \mathcal{E}_{k}(M)$ and metric $f$. Since $\mathbb{H}^{k} \subset \mathcal{E}_{k}(M)$ is convex, we can write $\mathbb{S}_{s}\left(\mathcal{E}_{k}(M)\right) \cap \mathbb{H}^{k}=\mathbb{S}_{s}\left(\mathbb{H}^{k}\right)$. Equivalently $\left(\mathbb{S}^{k+n-1} \times\{s\}\right) \cap \mathbb{H}^{k}=\mathbb{S}^{k-1} \times\{s\}$. Write $M_{o}=\{o\} \times M$. Also write

$$
E_{k}(M)=\mathcal{E}_{k}(M)-\left(\mathbb{H}^{k} \amalg M_{o}\right)
$$

and 


$$
S_{s}\left(\mathcal{E}_{k}(M)\right)=\mathbb{S}_{s}\left(\mathcal{E}_{k}(M)\right) \bigcap E_{k}(M)=\mathbb{S}_{s}\left(\mathcal{E}_{k}(M)\right)-\left(\mathbb{H}^{k} \amalg M_{o}\right)
$$

Note that the functions $\alpha$ and $\beta$ are well-defined and smooth on $E_{k}(M)$, and $0<\beta(p)<\pi / 2$. Moreover, by Remark 2.5, the plane $\mathbb{H}^{2}(p)=\gamma_{w} \times \eta_{u}$ is well defined for $p \in E_{k}(M)$. As in Remark 2.5 , here $p=(y, v) \in \mathbb{H}^{k} \times M, y=t w, v=r u$. Recall that $\triangle(p) \subset \mathbb{H}^{2}(p)$ (see Corollary 2.4 and Remark 2.5).

By the identification between $\mathbb{S}^{n+k-1} \times\{s\}$ with $\mathbb{S}_{s}\left(\mathcal{E}_{k}(M)\right)$ and Lemma 2.1 we have that $\mathbb{H}^{2}(p) \cap \mathbb{S}_{s}\left(\mathcal{E}_{k}(M)\right)$ gets identified with a geodesic circle $\mathbb{S}^{1}(p) \subset \mathbb{S}^{n+k-1}$. Moreover, since $\mathbb{H}^{2}(p)$ and $\mathbb{H}^{k}$ intersect orthogonally on $\gamma_{w}$, we have that the spherical geodesic segment $[x(p), w(p)]_{\mathbb{S}^{n+k-1}}$ intersects $\mathbb{S}^{k-1} \subset \mathbb{S}^{n+k-1}$ orthogonally at $w$. This together with the fact that $\beta<\pi / 2$ imply that $[x(p), w(p)]_{\mathbb{S}^{n+k-1}}$ is a length minimizing spherical geodesic in $\mathbb{S}^{k+n-1}$ joining $x$ to $w$. Consequently $\beta=\beta(p)$ is the length of $[x(p), w(p)]_{\mathbb{S}^{n+k-1}}$.

We now give a set of coordinates on $S_{s}\left(\mathcal{E}_{k}(M)\right)$. For $p \in S_{s}\left(\mathcal{E}_{k}(M)\right)$ define

$$
\Xi(p)=\Xi_{s}(p)=(w, u, \beta) \in \mathbb{S}^{k-1} \times \mathbb{S}^{n-1} \times(0, \pi / 2)
$$

where $w=w(p), u=u(p), \beta=\beta(p)$. Note that $\Xi$ is constant on geodesics emanating from $o \in \mathcal{E}_{k}(M)$, that is $\Xi(s x)=\Xi(x)$.

Using hyperbolic trigonometric identities (e.g. identity 2.6) we can find well defined and smooth functions $r=r(s, \beta)$ and $t=t(s, \beta)$ such that $r, s, t$ are the lengths of the sides of a right geodesic triangle on $\mathbb{H}^{2}$ with angle $\beta$ opposite the the side with length $r$. With these functions we can construct explicitly a smooth inverse to $\Xi$.

\subsection{Remarks.}

1. For $(w, u) \in \mathbb{S}^{k-1} \times \mathbb{S}^{n-1}$ we have

$$
\Xi\left(\left(\gamma_{w} \times \eta_{u}\right) \cap S_{s}\left(\mathcal{E}_{k}(M)\right)\right)=\{ \pm w\} \times\{ \pm u\} \times(0, \pi / 2)
$$

By Lemma 2.1 the paths $a \mapsto( \pm w, \pm u, a)$ four spherical (open) geodesic segments emanating orthogonally from $\mathbb{S}^{k-1}$.

2. For $w \in \mathbb{S}^{k-1}$ we have

$$
\Xi\left(\left(\gamma_{w} \times M\right) \cap S_{s}\left(\mathcal{E}_{k}(M)\right)\right)=\{ \pm w\} \times \mathbb{S}^{n-1} \times(0, \pi / 2)
$$

By Corollary 2.2 we have that this set is a spherical geodesic ball of radius $\pi / 2$ and of dimension $n$ (with its center deleted) intersecting $\mathbb{S}^{k-1}$ orthogonally at $w$. Note that the geodesic segments on this ball emanating from $w$ are the spherical geodesic segments of item 1 , for all $u \in \mathbb{S}^{n-1}$.

3. For $w \in \mathbb{S}^{k-1}$ and $r$ with $0<r<s$ we have

$$
\Xi\left(\left(\gamma_{w} \times \mathbb{S}_{r}(M)\right) \cap S_{s}\left(\mathcal{E}_{k}(M)\right)=\{w\} \times \mathbb{S}^{n-1} \times \beta(r)\right.
$$

where $\beta(r)$ is the angle of the right geodesic hyperbolic triangle with sides of length $s$ (opposite to the right angle) and $r$, opposite to $\beta$. By identity 2.6 we have $\beta=\sin ^{-1}\left(\frac{\sinh (r)}{\sinh (s)}\right)$.

4. Since the $M$-fibers $\{y\} \times M$ are orthogonal in $\mathcal{E}_{k}(M)$ to the $\mathbb{H}^{k}$-fibers $\mathbb{H}^{k} \times\{v\}$, items 1,2 , and 
3 above imply that the $\mathbb{S}^{k-1}$-fibers, the $\mathbb{S}^{n-1}$-fibers and $(0, \pi / 2)$-fibers are mutually orthogonal in $\mathbb{S}^{k-1} \times \mathbb{S}^{n-1} \times(0, \pi / 2)$ with the metric $\Xi_{*} f$.

5. The map

$$
\Xi^{\prime}=(\Xi, s): E_{k}(M) \rightarrow \mathbb{S}^{k-1} \times \mathbb{S}^{n-1} \times(0, \pi / 2) \times \mathbb{R}^{+}
$$

gives coordinates on $E_{k}(M)$.

\section{Section 4. Spherical Cuts on Hyperbolic Extensions.}

Let $\left(N^{m}, g\right)$ have center $o$. Recall from the Introduction that the metric $g_{r}$ on $\mathbb{S}_{r}$ is called the spherical cut of $g$ at $r$, and the metric $\hat{g}_{r}=\left(\frac{1}{\sinh ^{2}(r)}\right) g_{r}$ is the normalized spherical cut of $g$ at $r$.

Now let $\left(M^{n}, h\right)$ have center $o$. Thus we can write $h=h_{r}+d r^{2}$, where each $h_{r}$ is a metric on $\mathbb{S}^{n-1}$. As before we denote by $f=\mathcal{E}_{k}(h)$ the hyperbolic extension of $h$, and we write $f=f_{s}+d s^{2}$ on $\mathcal{E}_{k}(M)-\{o\}$; each $f_{s}$ is a metric on $\mathbb{S}^{n+k-1}$. We use the map $\Xi=\Xi_{s}$ of Section 3 that gives coordinates on $S_{s}\left(\mathcal{E}_{k}(M)\right)$. Note that the metric $\Xi_{*} f_{s}$ is a metric on $\mathbb{S}^{k-1} \times \mathbb{S}^{n-1} \times(0, \pi / 2)$, and it is the expression of $f_{s}$ in the $\Xi$-coordinates.

Proposition 4.1. The expression of $f_{s}$ in the $\Xi$-coordinates is given by

$$
\Xi_{*} f_{s}=\left(\sinh ^{2}(s) \cos ^{2}(\beta)\right) \sigma_{\mathbb{S}^{k-1}}+h_{r}+\left(\sinh ^{2}(s)\right) d \beta^{2}
$$

where $r=\sinh ^{-1}(\sinh (s) \sin (\beta))$ (see identity 2.6).

Remark 4.2. Note that the function $r=r(s, \beta)$ is the same function used in the Introduction for the $\theta$-reparametrizations $\lambda=\lambda\left(\lambda^{\prime}, \theta\right)$.

Proof. By Remark 3.1(4) we have that $\Xi_{*} f_{s}$ has the form $A+B+C$, where $A(u, \beta)$ is a metric on $\mathbb{S}^{k-1} \times\{u\} \times\{\beta\}, B(w, \beta)$ is a metric on $\{w\} \times \mathbb{S}^{n-1} \times\{\beta\}$ and $C(u, \beta)$ is a metric on $\{w\} \times\{u\} \times(0, \pi / 2)$, i.e. $C=f(w, u, \beta) d \beta^{2}$, for some positive function $f$.

Now, by definition we have

$$
f=\cosh ^{2}(r) \sigma_{\mathbb{H}^{k}}+h_{r}+d r^{2}=\cosh ^{2}(r)\left(\sinh ^{2}(t) \sigma_{\mathbb{S}^{k-1}}+d t^{2}\right)+h_{r}+d r^{2}
$$

By Proposition 2.7 and the identity $\cosh (r) \sinh (t)=\sinh (s) \cos (\beta)$ (which follows from the law of sines and the second law of cosines, also see identity 2.6$)$ we can write

$$
f_{s}+d s^{2}=f=\left(\sinh ^{2}(s) \cos ^{2}(\beta)\right) \sigma_{\mathbb{s}^{k-1}}+h_{r}+\left(\sinh ^{2}(s)\right) d \beta^{2}+d s^{2}
$$

This proves the proposition.

Hence Proposition 4.1 gives the expression of the spherical cut, at $s$, of the metric $f=\mathcal{E}_{k}(h)$ in the $\Xi$-coordinates. The next corollary does the same for the normalized spherical cut $\hat{f}$ of $f$ at $s$.

Corollary 4.3. The expression of $\hat{f}_{s}$ in the $\Xi$-coordinates is given by

$$
\Xi_{*}\left(\hat{f}_{s}\right)=\cos ^{2}(\beta) \sigma_{\mathbb{S}^{k-1}}+\sin ^{2}(\beta) \hat{h}_{r}+d \beta^{2}
$$


where $r$ as in Proposition 4.1.

Proof. Since $\sinh ^{2}(r) \hat{h}_{r}=h_{r}$, and $\sinh ^{2}(s) \hat{f}_{s}=f_{s}$, the corollary follows from Proposition 4.1 and identity 2.6.

\section{Section 5. Cut Limits and Proof of The Main Theorem.}

First a bit of notation. Let $\left(N^{m}, g\right)$ have center $o$. Recall that we can write the metric on $N-\{o\}=\mathbb{S}^{m-1} \times \mathbb{R}^{+}$as $g=g_{r}+d r^{2}$, where $r$ is the distance to $o$. Let $A \subset \mathbb{S}^{m-1}$ be open and denote by $\mathrm{C} A$ the open cone $A \times \mathbb{R}^{+} \subset \mathbb{S}^{m-1} \times \mathbb{R}^{+} \subset M$. We write $A_{r}=\mathrm{C} A \cap \mathbb{S}_{r}(M)=A \times\{r\}$. We say that $\left\{g_{\lambda}\right\}_{\lambda}$ is an $\odot$-family of metrics over $A$ if each $g_{\lambda}$ is a metric defined on $\mathrm{C} A$ and $g_{\lambda}$, and it can be written in the form $g_{\lambda}=\left(g_{\lambda}\right)_{r}+d r^{2}$ on $\mathrm{C} A$. We say that the $\left\{g_{\lambda}\right\}$ has cut limit over $A$, at $b$, if there is a $C^{2}$ metric $\hat{g}_{\infty}^{b}$ on $A$ such that (1.3) holds, where the arrow in (1.3) now means uniform convergence in the $C^{2}(A)$-norm on the space of $C^{2}$ metrics on $A \subset \mathbb{S}^{m-1}$. Also, cut limits over $A$, on $I$, and cut limits over $A$ are defined similarly.

Let $M^{n}$ have metric $h$ and center $o$. As always we identify $M-\{o\}$ with $\mathbb{S}^{n-1} \times \mathbb{R}^{+}$and $M$ with $\mathbb{R}^{n}$. Choose a center $o \in \mathbb{H}^{k} \subset \mathcal{E}_{k}(M)$. Let $\left\{h_{\lambda}\right\}_{\lambda}$ be a $\odot$-family of metrics on $M$, thus $o$ is a center for all $h_{\lambda}$. Denote by $f_{\lambda}=\mathcal{E}_{k}\left(h_{\lambda}\right)$ the hyperbolic extension of $h_{\lambda}$. We have that $\left\{f_{\lambda}\right\}_{\lambda}$ is a $\odot$-family on $\mathcal{E}_{k}(M)$. From now on we assume $\theta \in(0, \pi / 2]$ fixed. Next $\theta$-reparametrize $\left\{f_{\lambda}\right\}_{\lambda}$, that is, we use the change of variables $\lambda=\lambda\left(\lambda^{\prime}\right)=\sinh ^{-1}\left(\sinh \left(\lambda^{\prime}\right) \sin \theta\right)$. (Note that $\lambda^{\prime}$ plays the role of the variable $s$ in identity 2.6, and $\lambda$ plays the role of $r$.) We obtain in this way the $\odot$-family $\left\{f_{\lambda\left(\lambda^{\prime}\right)}\right\}_{\lambda^{\prime}}$. Write $S=\mathbb{S}^{n+k-1}-\left\{\mathbb{S}^{k-1} \amalg \mathbb{S}^{n-1}\right\}$, where $\mathbb{S}^{k-1} \subset \mathbb{H}^{k} \times\{o\}$ and $\mathbb{S}^{n-1} \subset\{o\} \times M$.

Proposition 5.1. Assume that $\left\{h_{\lambda}\right\}$ has cut limits on the interval $J_{c}=(-\infty, c]$, and that it is hyperbolic around the origin. Then for each $c^{\prime}<c+\ln \sin (\theta)$ the family $\left\{f_{\lambda_{\left(\lambda^{\prime}\right)}}\right\}_{\lambda^{\prime}}$ has cut limits on $J_{c^{\prime}}$ over $S$.

Proof. By hypothesis $\left\{h_{\lambda}\right\}$ is hyperbolic around the origin. Hence there is $B$ such that

$$
\widehat{\left(h_{\lambda}\right)_{\lambda+b}}=\sigma_{\mathbb{S}^{n-1}} \quad \text { for all } \quad b \leq B
$$

Hence the metrics $h_{\lambda}$ are canonically hyperbolic on the ball of radius $\lambda+B$. Also, since we are assuming $\left\{h_{\lambda}\right\}$ has cut limits on $J_{c}$ we have that

$$
b \in J_{c} \quad \Longrightarrow \quad{\widehat{\left(h_{\lambda}\right)}}_{\lambda+b} \stackrel{C^{2}}{\longrightarrow} \hat{h}_{\infty}^{b} \quad \text { as } \lambda \rightarrow \infty
$$

uniformly on $\mathbb{S}^{n-1}$ and uniformly with compact supports in the variable $b \in J_{c}$.

As mentioned before we can write $f_{\lambda}=\left(f_{\lambda}\right)_{s}+d s^{2}$. We have to compute the limit of $\widehat{\left(f_{\lambda\left(\lambda^{\prime}\right)}\right)_{\lambda^{\prime}+b}}$, as $\lambda^{\prime} \rightarrow \infty$. Let the $\Xi$-coordinates be as defined in Section 3 for the space $\left(\mathcal{E}_{k}(M), f\right)$.

From Corollary 4.3. we can express $\left(\hat{f}_{\lambda}\right)_{s}$ in $\Xi$-coordinates:

$$
\Xi_{*}\left(\widehat{\left(f_{\lambda\left(\lambda^{\prime}\right)}\right)_{\lambda^{\prime}+b}}\right)=\cos ^{2}(\beta) \sigma_{\mathbb{S}^{k-1}}+\sin ^{2}(\beta)\left(\widehat{h_{\lambda\left(\lambda^{\prime}\right)}}\right)_{r\left(\lambda^{\prime}+b, \beta\right)}+d \beta^{2}
$$


where $r=r(s, \beta)$ is given by identity 2.6 (see also Proposition 4.1 and Remark 4.2). Therefore we want to find the limit of $\left(\widehat{h_{\lambda\left(\lambda^{\prime}\right)}}\right)_{r\left(\lambda^{\prime}+b, \beta\right)}$ as $\lambda^{\prime} \rightarrow \infty$. To do this take the inverse of $\lambda=\lambda\left(\lambda^{\prime}\right)$, and we get $\lambda^{\prime}=\lambda^{\prime}(\lambda)=\sinh ^{-1}\left(\frac{\sinh (\lambda)}{\sin (\theta)}\right)$. Hence

$$
\left.\lim _{\lambda^{\prime} \rightarrow \infty} \widehat{\left(\widehat{h_{\lambda\left(\lambda^{\prime}\right)}}\right)_{r\left(\lambda^{\prime}+b, \beta\right)}}=\lim _{\lambda \rightarrow \infty} \widehat{\left(\widehat{\left.h_{\lambda}\right)}\right.}\right)_{\vartheta(\lambda, \beta, b)},
$$

where

$$
\vartheta(\lambda, \beta, b)=r\left(\lambda^{\prime}(\lambda)+b, \beta\right)=\sinh ^{-1}\left(\sinh \left\{b+\sinh ^{-1}\left(\frac{\sinh (\lambda)}{\sin (\theta)}\right)\right\} \sin (\beta)\right)
$$

and a straightforward calculation shows

$$
\lim _{\lambda \rightarrow \infty}(\vartheta(\lambda, \beta, b)-\lambda)=b+\ln \left(\frac{\sin (\beta)}{\sin (\theta)}\right) .
$$

This convergence is uniform with compact supports in the $C^{2}(S)$-topology (see caveat below). Choose $c^{\prime} \in \mathbb{R}$ such that $c^{\prime}<c-\ln \left(\frac{\sin (\pi / 2)}{\sin (\theta)}\right)=c+\ln \sin (\theta)$. Since $\beta \in(0, \pi / 2)$ we get

$$
b \in J_{c^{\prime}} \quad \Longrightarrow \quad\left(b+\ln \left(\frac{\sin (\beta)}{\sin (\theta)}\right)\right) \in J_{c} .
$$

Hence from (2), (3), (4) and (5) we get

$$
\lim _{\lambda^{\prime} \rightarrow \infty} \widehat{\left(\widehat{h_{\lambda\left(\lambda^{\prime}\right)}}\right)_{r\left(\lambda^{\prime}+b, \beta\right)}}=\hat{h}_{\infty}^{b+\ln \left(\frac{\sin \beta}{\sin \theta}\right)}
$$

Caveat. The limit (3) (hence also in (6)) is uniform with compact supports in the $\beta$ direction, but not uniform in the $\beta$ direction. The problem occurs when $\beta \rightarrow 0$.

We next deal with the problem mentioned in the caveat; that is, we have to show that the limit in (6) in uniform in the variable $\beta \in(0, \pi / 2)$ (not just uniform with compact supports). The convergence in (4) (hence in (6)) is uniform for $\beta$ near $\pi / 2$, but the convergence in (4) is certainly not uniform near 0 . Here is where we will need the extra condition of the family being hyperbolic near the origin. We will need the following claim.

Claim. Let $c, B, \theta \in \mathbb{R}$. Choose $c^{\prime}$ with $c^{\prime}<c+\ln \sin \theta$. Then there is $\beta_{1}>0$ such that $r\left(\lambda^{\prime}+c^{\prime}, \beta_{1}\right) \leq \lambda\left(\lambda^{\prime}\right)+B$, for every $\lambda^{\prime}$ sufficiently large.

Proof of the claim. A calculation shows that taking $\beta_{1}=\sin ^{-1}\left(e^{2(B-c-1}\right)$ works. (Find the limit $\lambda^{\prime} \rightarrow \infty$ of both terms in the inequality, and use the fact that $c^{\prime}<c+\ln \sin \theta$.) This proves the claim.

Since the function $r=r(s, \beta)$ is increasing in both variables, the claim implies that $r\left(\lambda^{\prime}+\right.$ $b, \beta) \leq \lambda\left(\lambda^{\prime}\right)+B$, for every $b \leq c^{\prime}, \beta \leq \beta_{1}$ and $\lambda^{\prime}$ sufficiently large (how large not depending on $b$, nor $\beta$ ). This together with (1) imply that for every $b \leq c^{\prime}, \beta \leq \beta_{1}$ and $\lambda^{\prime}$ sufficiently large we have

$$
\left.\widehat{\left(h_{\lambda\left(\lambda^{\prime}\right)}\right.}\right)_{r\left(\lambda^{\prime}+b, \beta\right)}=\sigma_{\mathbb{S}^{n-1}}
$$


Hence for every $b \in J_{c^{\prime}}$ and $\beta \leq \beta_{1}$ we have

$$
\lim _{\lambda^{\prime} \rightarrow \infty}\left(\widehat{h_{\lambda\left(\lambda^{\prime}\right)}}\right)_{r\left(\lambda^{\prime}+b, \beta\right)}=\sigma_{\mathbb{S}^{n-1}}
$$

Since $\beta_{1}>0$ the problem mentioned in the caveat (i.e. when $\beta \rightarrow 0$ ) has been removed. This proves the proposition.

Taking $c \rightarrow \infty$ in Proposition 5.1 gives the following corollary.

Corollary 5.2. Assume that $\left\{h_{\lambda}\right\}$ has cut limits, and that it is hyperbolic around the origin. Then $\left\{f_{\lambda\left(\lambda^{\prime}\right)}\right\}_{\lambda^{\prime}}$ has cut limits over $S$.

Proof of the Main Theorem. Note that the only difference between Corollary 5.2 and the Main Theorem is that in the corollary the cut limits exist over $S \subset \mathbb{S}^{n+k-1}$. Hence we have to show that the existence of cut limits over $S$ implies the existence of cut limits on the whole of $\mathbb{S}^{n+k-1}$. Corollary 5.2 and 1.3 in the Introduction imply

$$
\left|\left(\widehat{\left.\left(f_{\lambda}\right)\right|_{S}}\right)_{\lambda^{\prime}+b}-\hat{f}_{\infty}^{b}\right|_{C^{2}(S)} \longrightarrow 0 \quad \text { as } \quad \lambda^{\prime} \rightarrow \infty
$$

where $\hat{f}_{\infty}^{b}$ is a metric on $S$. In particular for every $b$ the one-parameter family $\left(\widehat{\left.\left(f_{\lambda}\right)\right|_{S}}\right)_{\lambda^{\prime}+b}$ is Cauchy, that is

$$
\left|\left(\left.\widehat{\left(f_{\lambda\left(\lambda_{1}^{\prime}\right)}\right)}\right|_{S}\right)_{\lambda_{1}^{\prime}+b}-\left(\widehat{\left(\left.\widehat{\left.f_{\lambda\left(\lambda_{2}^{\prime}\right)}\right)}\right|_{S}\right.}\right)_{\lambda_{2}^{\prime}+b}\right|_{C^{2}(S)} \longrightarrow 0
$$

uniformly on $S$ as $\lambda_{1}^{\prime}, \lambda_{2}^{\prime} \rightarrow \infty$. But since $S$ is dense in $\mathbb{S}^{n+k-1}$ we get that $\left.|g|_{S}\right|_{C^{2}(S)}=$ $|g|_{C^{2}\left(\mathbb{S}^{n+k-1}\right)}$, for any $C^{2}$ (pointwise) bilinear form $g$ on $\mathbb{S}^{n+k-1}$. Therefore we can drop the restriction " $\left.\right|_{S}$ " in $(7)$ to get

$$
\left.\mid \widehat{\left(f_{\lambda\left(\lambda_{1}^{\prime}\right)}\right)}\right)_{\lambda_{1}^{\prime}+b}-\left.\left.\widehat{\left(f_{\lambda\left(\lambda_{2}^{\prime}\right)}\right)}\right|_{\lambda_{2}^{\prime}+b}\right|_{C^{2}\left(\mathbb{S}^{n+k-1}\right)} \longrightarrow 0 \quad \text { as } \quad \lambda^{\prime} \rightarrow 0
$$

This implies that the family $\widehat{\left(f_{\lambda}\right)}{ }_{\lambda^{\prime}+b}$ is Cauchy. Since the space of $C^{2}$ metrics on $\mathbb{S}^{n+k-1}$ with the $C^{2}$ norm is a complete metric space the Cauchy sequence above converges to some $\hat{f}_{\infty}^{b}$. Note that $\hat{f}_{\infty}^{b}$ is a symmetric bilinear form on $\mathbb{S}^{n+k-1}$, and it is positive definite on $S$. It remains to prove that $\hat{f}_{\infty}^{b}$ is also positive definite outside $S$. Recall $S=\mathbb{S}^{n+k-1}-\left(\mathbb{S}^{k-1} \amalg \mathbb{S}^{n-1}\right)$. But it is straightforward to verify that we have $\left.\hat{f}_{\infty}^{b}\right|_{\mathbb{S}^{k-1}}=\sigma_{\mathbb{S} k-1}+\sigma_{\mathbb{H}^{n}}$. On the other hand on $\mathbb{S}^{n-1}$ we have $\beta=\pi / 2$, hence $\lambda=\lambda^{\prime}$. Also by definition we have $f_{\lambda}=\cosh ^{2}(r) \sigma_{\mathbb{H} k}+h_{\lambda}$. But on $M_{o}$ we get $r=s$. Therefore

$$
\left(\left(\widehat{\left.f_{\lambda}\right)\left.\right|_{\mathbb{S}^{n-1}}}\right)_{\lambda^{\prime}+b}=\left(\left(\widehat{\left.f_{\lambda}\right)\left.\right|_{\mathbb{S}^{n-1}}}\right)_{\lambda+b}=\operatorname{cotanh}^{2}(\lambda+b) \sigma_{\mathbb{H}^{k}}+\widehat{\left(h_{\lambda}\right)}\right)_{\lambda+b} \longrightarrow \operatorname{cotanh}^{2}(\lambda+b) \sigma_{\mathbb{H}^{k}}+\hat{h}_{\infty}^{b}\right.
$$

Consequently $\hat{f}_{b+\infty}$ is positive definite on $\mathbb{S}^{n-1}$. Thus it is positive definite outside $S$. This proves the Main Theorem. 


\section{References}

[1] R.L. Bishop and B. O'Neill, Manifolds of negative curvature, Trans. Amer. Math. Soc. 145 (1969) 1-49.

[2] R. M. Charney and M. W. Davis, Strict hyperbolization, Topology 34 (1995), 329-350.

[3] P. Ontaneda, Riemannian Hyperbolization. Arxiv: 1406.1730.

[4] P. Ontaneda, Hyperbolic extensions and metrics $\epsilon$-close to hyperbolic. Arxiv: 1406.1740. To appear in Indiana Univ. Math. J.

[5] P. Ontaneda, Deforming an $\epsilon$-close to hyperbolic metric to a hyperbolic metric. Arxiv: 1406.1743. Submitted for publication.

Pedro Ontaneda

SUNY, Binghamton, N.Y., 13902, U.S.A. 